\title{
Local versus systemic treatment intensification: what is the optimal strategy for localized prostate cancer?
}

(c) The Author(s), under exclusive licence to Springer Nature Limited 2022

Prostate Cancer and Prostatic Diseases (2022) 25:7-8; https://doi. org/10.1038/s41391-022-00505-w

In clinical trials spanning several decades, the two most common intensification strategies investigated for improving outcomes in patients with localized prostate cancer undergoing definitive radiotherapy (RT) have been androgen deprivation therapy (ADT) and radiation dose-escalation. Although both strategies have been adopted as standard components of modern therapeutic paradigms for prostate cancer, they have divergent impacts on clinical outcomes. Randomized trials have consistently shown improved overall survival, prostate cancer specific mortality (PCSM), and distant metastasis (DM) rates when comparing combined ADT and radiation to radiation alone in intermediate- and high-risk prostate cancer [1-4], whereas dose-escalation trials have largely shown improved PSA recurrence rates only, without survival improvements [5]. Despite this, for years there have been those who have argued that dose-escalation can abrogate the need for ADT in prostate cancer treatment.

To try to clarify the relative benefits of ADT and dose-escalation in prostate cancer, Jiang and colleagues analyzed 40 randomized trials enrolling 21,429 patients with prostate cancer undergoing definitive radiation with median follow-up of 9.2 years [6]. Five different treatment strategies were compared: (1) low-dose RT alone ( $\leq 74 \mathrm{~Gy}),(2)$ high-dose RT alone (>74 Gy), (3) low-dose RT with short-term ADT, (4) low-dose RT with long-term ADT, and (5) high-dose RT with short-term ADT. Given so many possible pairwise comparisons of these five treatment strategies, interpretation of the results is somewhat complex, and multiple hypothesis corrections were applied. The following are some takeaways from the reported comparisons. First, no differences in PCSM or DM were observed with dose-escalation, either in patients treated with RT alone or RT with short-term ADT. Second, in patients treated low-dose RT, ADT was associated with improved PCSM and DM. Third, there was no associated in improvement in PCSM with short-term ADT and dose-escalated RT versus dose-escalated RT alone (although there was lower PCSM associated with long-term ADT and low-dose RT versus high-dose RT alone). Last, none of the treatment arms was associated with differences in overall survival after correcting for multiple hypothesis testing.

The authors should be commended for executing a thorough analysis of the available data. It is hard to argue with the authors' conclusion that the benefit of adding ADT to low-dose RT outweighs the benefit of dose-escalation based on the totality of prior evidence to date. However, the limitations of the methodology of this particular study preclude making any strong further conclusions or trying to parse the data too finely. Although the data analyzed are derived from randomized trials, the comparisons presented are not aggregated randomized comparisons, as is common in many metaanalyses, but rather are cross-trial comparisons of grouped individual arms from various trials. This largely eliminates any balancing of measured and unmeasured confounders resulting from randomization, making the comparisons prone to bias. This is further exacerbated by the fact that these comparisons involve trials that span more than two decades during an era that has seen significant stage migration as a result of Gleason score inflation and increased imaging utilization. As an illustrative example, the low-dose RT alone group includes patients from RTOG 8531 (100\% high-risk, enrollment midpoint 9/1989) [3], and whereas the high-dose RT group includes both arms of PROFIT ( $0 \%$ high-risk, enrollment midpoint 1/2008) [7]. Thus, although outcome differences in the treatment groups could be in part due to the treatments delivered, they are unquestionably also a result of completely different populations and eras of the trials involved. To their credit, the authors adjusted comparisons for median age, midpoint of study enrollment, and proportion of high-risk patients. However, given the lack of individual patient data, there can only be fairly rudimentary adjustments, and more refined balancing of confounding factors is not possible.

Ultimately, there is no question that the preponderance of the evidence supports ADT as having a stronger influence on metastasis and PCSM than dose-escalation in unfavorable-risk prostate cancer. Thus, dose-escalation cannot abrogate the need for ADT without prospective randomized evidence. Rather than focusing on the specific radiation dose employed, studies that aim to more accurately identify patients with high enough absolute risk of metastasis and PCSM to derive a clinically meaninful benefit from ADT, such as the forthcoming NRG GU010 GUIDANCE trial (NCT05050084), likely represent a more promising strategy to optimize the risks and benefits of ADT in prostate cancer patients undergoing definitive RT.

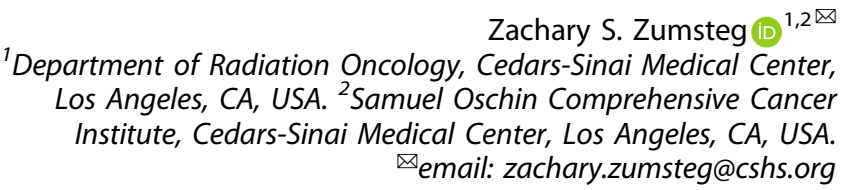

\section{REFERENCES}

1. Jones CU, Hunt D, McGowan DG, Amin MB, Chetner MP, Bruner DW, et al. Radiotherapy and short-term androgen deprivation for localized prostate cancer. N Engl J Med. 2011;365:107-18.

2. D'Amico AV, Chen MH, Renshaw AA, Loffredo M, Kantoff PW. Androgen suppression and radiation vs radiation alone for prostate cancer: a randomized trial. JAMA.2008;299:289-95. 
3. Pilepich MV, Winter K, Lawton CA, Krisch RE, Wolkov HB, Movsas B, et al. Androgen suppression adjuvant to definitive radiotherapy in prostate carcinomalong-term results of phase III RTOG 85-31. Int J Radiat Oncol Biol Phys. 2005;61:1285-90.

4. Bolla M, Van Tienhoven G, Warde P, Dubois JB, Mirimanoff RO, Storme G, et al. External irradiation with or without long-term androgen suppression for prostate cancer with high metastatic risk: 10-year results of an EORTC randomised study. Lancet Oncol. 2010;11:1066-73.

5. Zaorsky NG, Keith SW, Shaikh T, Nguyen PL, Horwitz EM, Dicker AP, et al. Impact of radiation therapy dose escalation on prostate cancer outcomes and toxicities. Am J Clin Oncol. 2018;41:409-15.

6. Jiang T, Markovic D, Patel J, Juarez JE, Ma TM, Shabsovich D, et al. Radiation therapy dose and androgen deprivation therapy in localized prostate cancer: a meta-regression of 5-year outcomes in phase III randomized controlled trials. Prostate Cancer Prostatic Dis. 2021. https://doi.org/10.1038/s41391-02100432-2.

7. Catton CN, Lukka H, Gu CS, Martin JM, Supiot S, Chung PWM, et al. Randomized trial of a hypofractionated radiation regimen for the treatment of localized prostate cancer. J Clin Oncol. 2017;35:1884-90.

\section{AUTHOR CONTRIBUTIONS}

$\mathrm{ZZ}$ is the sole author for this work.

\section{COMPETING INTERESTS}

Dr. Zumsteg's spouse does legal work for Johnson \& Johnson and Allegan. There are no other conflicts of interest to disclose.

\section{ADDITIONAL INFORMATION}

Correspondence and requests for materials should be addressed to Zachary $\mathrm{S}$. Zumsteg.

Reprints and permission information is available at http://www.nature.com/ reprints

Publisher's note Springer Nature remains neutral with regard to jurisdictional claims in published maps and institutional affiliations. 